\title{
Caracterización biométrica y estimación del peso corporal en bovinos criollos en la comunidad de Chuñuranra-Huancavelica (Perú)
}

\author{
Contreras, J.L1@.; Cordero, A.G ${ }^{3}$; Curasma, J. ' ; Enriquez, D'; Vilcapaza, L. ${ }^{2}$;Guitierrez N. ${ }^{1}$ y Del Solar, J. ${ }^{4}$
}

\author{
'Laboratorio de nutrición animal y evaluación de alimentos: Facultad Ciencias de Ingeniería: E.P Zootecnia. UNH. Huancavelica, Perú. \\ 2Laboratorio de Sanidad Animal: Facultad Ciencias de Ingeniería: E.P Zootecnia. UNH. Huancavelica, Perú. \\ ${ }^{3}$ Docente de la Escuela de Post-Grado de la Facultad de Ciencias de Ingeniería UNH, Perú. \\ 4Programa de Pós-graduação em Zootecnia, Universidade Federal da Bahía, Brasil.
}

\section{PALABRAS CLAVE}

Biometría.

Caracterización.

Interrelaciones productivas.

Vacunos criollos.

\section{RESUMEN}

96 bovinos criollos, procedentes del distrito de Huancavelica (Perú), de edades y sexos diferentes fueron estudiados con el objetivo de determinar correlaciones fenotípicas entre el peso corporal $(\mathrm{PC})$ con las medidas biométricas: Ancho de la cabeza (AC) y longitud de la cabeza (LC); ancho de tórax (ATO), longitud de tórax (LT), perímetro de tórax (PT) y altura de tórax (ALT); ancho de la grupa (AGR), longitud de la grupa (LGR) y altura de la grupa (AG), distancia dorso lumbar (DL); longitud (LCU) y longitud total de cuerpo (TO), altura de cruz (ACR) y perímetro abdominal (PA). Estas características fueron ajustadas en una regresión lineal múltiple de primer grado para obtener una ecuación de predicción de PC. Las correlaciones fenotípicas fueron altas y significativas ( $p<0.001$ ) entre PC-AG (0.57), PC-PT (0.58), PCLAC (0.60), PC-ACR (0.56) y PC-PA (0.58). La predicción de PC puede hacerse mediante la ecuación: $P C=-321.043+1.82 P A+0.78928 L A C+1.46924 A C R . P T, L A C$ y ACR pueden ser útiles y servir como buenos indicativos del PC de los bovinos criollos.

\section{Biometric characterization and estimation of body weight in creole cattle in the community of Chunuranra - Huancavelica (Perú)}

\begin{abstract}
SUMMARY
96 creole cattle from Huancavelica region (Peru) of different ages and sexes were studied aiming to determinate phenotypic correlations between body weight (PC) and biometric measurements: Width (HW) and head length $(\mathrm{HL})$; width $(\mathrm{ChW})$, length $(\mathrm{ChL})$, perimeter $(\mathrm{ChP})$ and chest height $(\mathrm{ChH})$; width $(\mathrm{CrW})$, length $(\mathrm{CrL})$ and croup height $(\mathrm{Cr} H)$, back lumbar distance (BLD); length $(\mathrm{BL})$ and full body length (FBW), cross height ( $\mathrm{CrOH}$ ) and abdominal perimeter (AP). These traits were adjusted in a first-degree multiple linear regression to obtain a $\mathrm{PC}$ prediction equation. Phenotypic correlations were high and significant $(\mathrm{p}$ $<0.001$ ) between PC-AG (0.57), PC-PT (0.58), PC-LAC (0.60), PC-ACR (0.56) and PC-PA (0.58). The PC prediction can be done by the equation: $\mathrm{PC}=-321.043+1.82 \mathrm{AP}+0.78928 \mathrm{BL}+1.46924 \mathrm{CroH} . \mathrm{AP}$, $\mathrm{BL}$ and $\mathrm{CroH}$ can be useful and serve as good indicative of $\mathrm{PC}$ of the creole cattle.
\end{abstract}

\section{INFORMATION}

Cronología del artículo.

Recibido/Received: 06.07.2018

Aceptado/Accepted: 23.06.2021

On-line: 15.07.2021

Correspondencia a los autores/Contact e-mail:

joselcpunh123@hotmail.com

\section{INTRODUCCIÓN}

En la sierra peruana, la ganadería está constituida por fauna autóctona (alpacas, vicuñas, llamas, guanacos) y por animales introducidos por los españoles en el siglo XVI en la que destacan vacunos, ovinos, porcinos, caprinos y aves (Flores 2002, p. 246). En Huancavelica la población actual de ganado vacuno es de 5156, la cual es mayor en 14,7\% a la registrada en el censo agropecuario de 1994. La raza predominante en esta región es la criolla, representando el 63,9\% de la población de vacunos en el Perú (CENAGRO 2012, p. 16).

El ganado criollo se destaca en este país como productor de carne y fuerza de trabajo. Su producción de leche no es importante aun cuando produce entre 450 a 1500 litros de leche por campaña. Sin embargo, este genotipo adaptado presenta un potencial lechero si se dan las condiciones de alimentación, sanidad y manejo adecuados. A la producción de carne contribuyen las hembras al final de su vida productiva, sea la hembra como productora de terneros o de leche; y los 
machos, como reproductores o unidades de tracción, pasando a ser sacrificados directamente o vendidos para ser engordados (Flores 2002, p. 280).

La genética base de estos animales permitiría la generación de nuevos genotipos especializados en producción de leche, carne o doble propósito, conservando sus características de adaptación y habilidad materna (Florio, 2008, p. 124). Por lo tanto, el ganado criollo en el Perú tiene gran importancia como pie de cría o base, sobre la cual se pueden mejorar genéticamente algunas características de interés económico, conservando su rusticidad y adaptación a climas alto-andinos (Flores, 1993, p. 70). Además, es necesaria su preservación garantizando su diversidad genética, mediante cruzamientos dirigidos ó inclusive la generación de razas sintéticas locales (Cevallos et al. 2013, p. 16).

Las medidas biométricas son utilizadas en la estimación del peso vivo en diversas especies y razas (Ribeiro et al., 2000, p. 12). Del mismo modo, la caracterización fenotípica de parámetros morfológicos en los animales, genera información útil para la predicción del peso en todas las edades (Osorio et al., 1997, p. 267). Esto puede ser obtenido mediante correlaciones entre diversas medidas biométricas y regresiones múltiples, cuyo objetivo es obtener ecuaciones con el poder de predicción de peso (Matos et al., 1978, p. 233). En el Perú se dispone de escasa información sobre la relación entre variables productivas en ganado bovino criollo. Por esta razón, este trabajo busca relacionar el peso corporal (PC) con otras medidas biométricas y definir su ecuación de predicción en bovinos criollos criados en la región de Huancavelica, Perú.

\section{MATERIAL Y MÉTODOS}

\section{LUGAR DEL ESTUDIO}

Las medidas biométricas de los animales se registraron durante los meses de noviembre y diciembre del 2017, en el Camal Municipal de Huancavelica (12 ${ }^{\circ} 46^{\prime} 57^{\prime \prime}$ latitud sur; $74^{\circ} 58^{\prime} 21^{\prime \prime}$ longitud oeste) a 3704 metros de altitud con temperaturas anual máxima de $16^{\circ} \mathrm{C}\left(61^{\circ} \mathrm{F}\right)$ y mínima de $2^{\circ} \mathrm{C}\left(36^{\circ} \mathrm{F}\right)$. La temporada seca comprende los meses de mayo a octubre y la temporada lluviosa, los meses de noviembre a abril), con precipitación de $784 \mathrm{~mm}$ por año.

Las medidas biométricas de los animales se registraron durante los meses de noviembre y diciembre del 2017, en el Camal Municipal de Huancavelica (12 $46^{\prime} 57^{\prime \prime}$ latitud sur; $74^{\circ} 58^{\prime} 21^{\prime \prime}$ longitud oeste) a 3704 metros de altitud con temperaturas anual máxima de $16^{\circ} \mathrm{C}\left(61^{\circ} \mathrm{F}\right)$ y mínima de $2^{\circ} \mathrm{C}\left(36^{\circ} \mathrm{F}\right)$. La temporada seca comprende los meses de mayo a octubre y la temporada lluviosa, los meses de noviembre a abril), con precipitación de $784 \mathrm{~mm}$ por año.

\section{DistribuCIÓN DE lOS ANIMALES EN ESTUDIO.}

En el presente estudio, fueron evaluados 96 bovinos criollos separados en categorías de acuerdo a su edad y sexo, ver Tabla I. La mayor parte de los animales fueron hembras $(79.16 \%)$. Los animales de boca llena participaron con el $35.41 \%$, seguidos por los de cuatro dientes $(20.83 \%)$ y en menor cantidad los animales de seis dientes (7.29 \%). El sistema de crianza de los animales del presente estudio es exten- sivo a base de pastizales constituidos principalmente de especies deseables (51.20\%): Alchemilla pinnata,

Tabla I. Distribución absoluta y porcentual de los bovinos Criollos por edad dentaria y sexo (Absolute and percentage distribution of Criollo cattle by tooth age and sex).

\begin{tabular}{lllllll}
\hline Edad & $\begin{array}{l}\text { Sexo } \\
\text { Machos }\end{array}$ & $\%$ & Hembras & $\%$ & Total & $\%$ \\
& & & & & & \\
DL & 9 & 45.00 & 7 & 9.21 & 16 & 16.67 \\
2D & 5 & 25.00 & 14 & 18.42 & 19 & 19.79 \\
4D & 1 & 5.00 & 19 & 25.00 & 20 & 20.83 \\
6D & 1 & 5.00 & 6 & 7.89 & 7 & 7.29 \\
BL & 4 & 20.00 & 30 & 39.47 & 34 & 35.42 \\
Total & 20 & 20.83 & 76 & 79.16 & 96 & 100 \\
\hline
\end{tabular}

DL: Dientes de leche; 2D: 2 dientes; 4D: 4 dientes; 6D: 6 dientes; BL: Boca llena

Festuca dolichophylla y Disticha muscoides; poco deseables (42.00\%): Poa alquigluma, Calamagrostis ovata y Calamagrostis curvula, e indeseables (6.80\%): Aciachne pulvinata, Plantago rígida y Stipa obtusa (Quinto, 2004, p. 24). Algunos productores utilizaban heno de avena, como alimentación suplementaria.

\section{VARIABLE BIOMÉTRICA EN ESTUDIO.}

Siguiendo la metodología descrita por Rojas y Gómez (2005, p. 235), con báscula ganadera de $1000 \mathrm{~kg}$ de capacidad, se midieron las siguientes variables biométricas: peso corporal (PC); ancho de cabeza (AC), tomada por debajo de los ojos partiendo de los ángulos faciales, con el compás; longitud de cabeza (LC), tomada con compás entre el occipucio (parte media) y la punta del morro; ancho de tórax (ATO), medida entre los puntos de encuentro derecho e izquierdo; ancho de grupa (AGR), medida entre la punta de nalga y tuberosidad isquiática; longitud de grupa (LGR), medida entre la tuberosidad coxal y la punta de nalga; distancia dorso lumbar (DL), medida entre la inserción de la cola hasta la cruz; altura de tórax (ALT), medida de la cruz a la cinchera hasta el codo del miembro anterior; altura de la grupa (AG), medida entre el nivel de la punta de anca hasta el nivel del piso; longitud de tórax (LT), medida desde el punto de encuentro hasta mitad de la última costilla falsa; perímetro torácico $(\mathrm{PT})$, tomada con cinta métrica alrededor del tórax; longitud de cuerpo (LAC), medida desde el testuz hasta la inserción de la cola; longitud total (LCU) medida desde el punto de encuentro hasta punta de nalga; altura de cruz (ACR) o alzada , medida con hipómetro desde la base del piso a la cruz; perímetro abdominal (PA), medida con cinta métrica alrededor del abdomen a nivel de la cicatriz umbilical.

Los resultados fueron analizados mediante el análisis de varianza para determinar el efecto del sexo y la edad y su interacción, sobre las medidas biométricas.

\section{ANÁLISIS ESTADÍSTICO}

Se determinaron las correlaciones fenotípicas entre el PC y las demás medidas biométricas, con al procedimiento CORR del SAS (2009), versión 9.2. 
De acuerdo a lo descrito por (Ribeiro et al. 2004, p. 338) fueron consideradas los coeficientes de correlación de Pearson mayores entre el PC y las características biométricas en estudio, para ser sometidas a un análisis de regresión lineal múltiple de primer grado a través del procedimiento REG del SAS (2009, con el siguiente modelo: Pcij $=\mathrm{u}+\mathrm{bnXi}+\mathrm{e}(\mathrm{ij})$, en donde: PCij es peso corporal de los bovinos criollos; bn son los coeficientes de regresión de las Xi características incluidas en el modelo, finalmente eij es el efecto residual aleatorio asociado a PC en cada individuo.

\section{RESULTADOS Y DISCUSIONES}

\section{PRESENTACIÓN DE LOS RESULTADOS Y DISCUSIÓN}

En la Tabla II se muestran las medidas biométricas de los bovinos criollos. La AG, PT, ACR y PA presentaron variabilidad baja, entre $7.21 \%$ y $9.75 \%$. Estas variabilidades guardan relación con lo relatado por Cevallos et al. (2013, p. 17), excepto para el PA. Para PC, AC, LC, ATO, AGR, LGR, DL, ALT, LT, LAC y LCU fueron observadas variabilidades moderadas (10.11 \% y $19.56 \%$ ), valores que están por encima de lo obtenido por Cevallos et al. (2013, p. 17). Esto puede indicar que existe una variación en esta población, lo cual puede ser favorable para ser utilizados en programas de mejoramiento.

Correlaciones fenotípicas de las características biométricas.

De las 1344 mediciones realizadas en 96 bovinos Criollos resultaron 105 correlaciones entre las diferentes características. De ellas se establecieron las mayores correlaciones entre el PC y las medidas biométricas: AG, PT, LAC, ACR y PA (Tabla IV), fueron las características biométricas que demostraron correlaciones altas y positivas $(\mathrm{p}<0.01)$ con el $\mathrm{PC}$, siendo las más indicadas para estimar el PC en ambos sexos y con edades comprendidas entre dientes de leche y boca llena. Se observan que las correlaciones PC-PT, PC-LAC y PC-ACR, son muy inferiores a lo observado por Mahecha et al. (2002a ${ }^{\mathrm{a}}$, p. 91) en la raza Lucerna, con valores que oscilan entre 0.94 y 0.97. Muñoz (2002, p. 126), indica si el tamaño de muestra es pequeño, el coeficiente de correlación (r) es una estimación ligeramente parcial y subestima el coeficiente de correlación poblacional $(\rho)$ en pequeñas muestras. Hecho que probablemente haya ocurrido en el presente estudio, pues se trató de variables aleatorias bivariadas. Valores iguales de correlación $(\mathrm{r}=0.58, \mathrm{P}<0.001)$ se encontraron entre el PC - PT y PC - PA, indicando que entre los bovinos de mayor PT se encuentran animales con mayor PC. Otros reportes muestran correlaciones entre el PC y el PT más altas que las observadas en el presente estudio (Hernández et al., 1984, p. 45, Menéndez et al., 1987, p. 15 y Henao y Mejia, 1994, p. 79), en terneros machos Cebú, ganado Cebú y Pardo Suizo, respectivamente. Esto indica que tales características son propias de ganado productor de carne, colocandolas como criterio de selección en esquemas de selección.

\section{LA ECUACIÓN PARA LA ESTIMACIÓN DEL PESO VIVO.}

La ecuación de regresión múltiple reveló influencia $(\mathrm{p}<0.01)$ de PT, LAC y ACR en PC de bovinos criollos, cuya ecuación ajustada es PC $=-321.04315+$ $1.82146 \mathrm{PT}+0.78928 \mathrm{LAC}+1.46924 \mathrm{ACR}(\mathrm{R} 2=0.6143)$. Este modelo indica que, por la variación de un centí-

Tabla II. Estadística descriptiva para peso corporal $(\mathrm{kg})$ y características biométricas (cm) de bovinos criollos criados en sistema extensivo en zona alto andina (Descriptive statistics for body weight $(\mathrm{kg})$ and biometric characteristics $(\mathrm{cm})$ of Creole bovines raised in an extensive system in the Andean highlands).

\begin{tabular}{|c|c|c|c|c|c|}
\hline Característica & Media & Mínimo & Máximo & $\mathrm{DE}$ & $\mathrm{CV}(\%)$ \\
\hline$P C(k g)$ & 234.51 & 74.00 & 367.00 & 59.37 & 19.56 \\
\hline $\mathrm{AC}(\mathrm{cm})$ & 18.87 & 14.00 & 25.00 & 2.37 & 10.19 \\
\hline $\mathrm{LC}(\mathrm{cm})$ & 41.75 & 26.00 & 53.60 & 4.64 & 10.11 \\
\hline ATO $(\mathrm{cm})$ & 37.88 & 23.00 & 60.10 & 8.67 & 19.35 \\
\hline AGR $(\mathrm{cm})$ & 35.48 & 15.00 & 48.60 & 8.03 & 18.23 \\
\hline LGR (cm) & 37.00 & 26.00 & 48.00 & 5.94 & 15.94 \\
\hline $\mathrm{DL}(\mathrm{cm})$ & 102.18 & 17.00 & 132.00 & 16.25 & 14.77 \\
\hline $\operatorname{ALT}(\mathrm{cm})$ & 54.39 & 37.00 & 67.60 & 7.17 & 10.84 \\
\hline $\mathrm{AG}(\mathrm{cm})$ & 114.21 & 92.10 & 143.00 & 9.40 & 7.21 \\
\hline $\mathrm{LT}(\mathrm{cm})$ & 64.24 & 44.00 & 87.00 & 10.03 & 14.50 \\
\hline $\mathrm{PT}(\mathrm{cm})$ & 151.13 & 102.00 & 183.00 & 14.31 & 7.35 \\
\hline $\mathrm{LAC}(\mathrm{cm})$ & 149.63 & 95.00 & 188.00 & 21.01 & 12.43 \\
\hline $\operatorname{LCU}(\mathrm{cm})$ & 121.71 & 61.00 & 183.00 & 21.42 & 16.11 \\
\hline $\mathrm{ACR}(\mathrm{cm})$ & 110.38 & 90.00 & 139.00 & 9.81 & 7.46 \\
\hline $\mathrm{PA}(\mathrm{cm})$ & 162.65 & 109.50 & 210.00 & 18.30 & 9.75 \\
\hline
\end{tabular}

$\mathrm{PC}=$ peso corporal; $\mathrm{AC}=$ ancho de cabeza; $\mathrm{LC}=$ longitud de cabeza; $\mathrm{ATO}=$ ancho de tórax; $\mathrm{AGR}=$ ancho de grupa; $\mathrm{LGR}=$ longitud de grupa; $\mathrm{DL}=$ distancia dorso lumbar; $\mathrm{ALT}$ = altura de tórax; $\mathrm{AG}=$ altura de la grupa; $\mathrm{LT}=$ longitud de tórax; $\mathrm{PT}=$ perímetro torácico; $\mathrm{LAC}=$ largo de cuerpo; LCU = longitud de cuerpo; ACR = altura de cruz o alzada; PA = perímetro abdominal. 


\begin{abstract}
Tabla IV. Coeficientes de correlación de Pearson (r) entre el peso corporal (PC) y características biométricas en bovinos criollos criados en sistema extensivo en zona altoandina (Pearson correlation coefficients ( $r$ ) between body weight (BW) and biometric characteristics in Creole cattle raised in an extensive system in the high Andean zone).
\end{abstract}

\begin{tabular}{llll}
\hline & & \multicolumn{2}{l}{ Correlación de Pearson } \\
PC $1 ~$ & C2 & $r$ & $p-$ valor \\
& AC & 0.34 & $* * *$ \\
& LC & 0.19 & ns \\
& ATO & 0.45 & $* * *$ \\
& AGR & 0.50 & $* * *$ \\
LGR & 0.09 & ns \\
DL & 0.45 & $* * *$ \\
ALT & 0.47 & $* * *$ \\
AG & 0.57 & $* * *$ \\
LT & 0.37 & $* * *$ \\
PT & 0.58 & $* * *$ \\
LAC & 0.60 & $* * *$ \\
LCU & 0.38 & $* * *$ \\
ACR & 0.56 & $* * *$ \\
PA & 0.58 & $* * *$ \\
\hline
\end{tabular}

C1 = Característica 1; C2 = Característica 2; PC = peso corporal; $A C=$ ancho de cabeza; $L C=$ longitud de cabeza; $A T O=$ ancho de tórax; $A G R$ = ancho de grupa; $L G R$ = longitud de grupa; $D L$ = distancia dorso lumbar; $A L T$ = altura de tórax; $A G$ = altura de la grupa; $\mathrm{LT}=$ longitud de tórax; $\mathrm{PT}=$ perímetro torácico; $\mathrm{LAC}=$ largo de cuerpo; $\mathrm{LCU}=$ longitud de cuerpo; $A C R=$ altura de cruz o alzada; $\mathrm{PA}=$ perímetro abdominal. NS $=$ No significativo; ${ }^{*},{ }^{* *} \mathrm{y} * * *$ significativo cuando $p<0.05, p<0.01$ y $p<0.001$, respectivamente.

metro del PT, de LAC y de ACR; el PC es modificado $1.82,0.79$ y $1.47 \mathrm{~kg}$, respectivamente.

En este modelo, el PT ocupa la primera posición, seguido de LAC y ACR. Mahecha et al. (2002a, p. 90) en la raza Lucerna, relatan el orden de prioridad de mediciones corporales con relación a los mayores coeficientes de correlación, en hembras perímetro torácico, longitud corporal y altura corporal, ya en machos serían perímetro torácico, altura corporal y longitud corporal, en ese orden. Esto demuestra que el PT es la medida biométrica que más se ha utilizado en la predicción del peso vivo de los bovinos (Mahecha et al., 2002b, p. 83).

\section{CONCLUSIONES}

Las medidas biométricas estudiadas proporcionaron buenos indicativos del peso corporal de los bovinos criollos, pero con cierta limitación, por la presencia de correlaciones moderadas.

Las variables perímetro torácico, largo de cuerpo y altura de cruz o alzada fueron las mejores medidas relacionadas funcionalmente con el peso corporal.

\section{AGRADECIMIENTOS}

Se agradece al Fondo de Desarrollo Socio Económico del Proyecto de Camisea-FOCAM del proyecto de siembra de avena y cebada por el apoyo con equipos (bascula) y al Fondo de Incentivos en Apoyo a la Investigación- FAEDI de la Universidad Nacional de Huancavelica por el apoyo económico, y a los ganaderos dedicados a la crianza de ganado bovino criollo de la comunidad de Chuñuranra - Huancavelica por haber cedido sus animales para la toma de las medidas biométricas.

\section{BIBLIOGRAFÍAS}

Cevallos, FO, Estupiñán, VK y Torres 2013," Caracterización morfoestructural y faneróptica del bovino Criollo de la provincia de Manabí, Ecuador. XXII Reunión de la Asociación Latinoamericana de Producción Animal-ALPA". IV Congreso Internacional de Producción Animal Tropical. Habana, Cuba, pp. 12-16.

CENAGRO 2012, "IV Censo Nacional Agropecuario", Disponible en: http://proyectos.inei.gob.pe/web/DocumentosPublicos/ResultadosFinalesIVCENAGRO.pdf. Acceso: 12 de abril del 2018, vol, p 63.

Flores, A 2002, "Visión panorámica de la ganadería. En: Caballero W, Flores A, Arroyo O, Alcántara A (eds). Hacia una nueva agricultura, con énfasis en la generación y transferencia de tecnología", Lima: CONCYTEC, p 245-314.

Flores, A 1993, "Producción de ganado vacuno de carne-Mejoramiento. In Rosemberg, M. Manual de producción de ganado vacuno de carne. Proyecto de Transformación de la Tecnología Agropecuaria", Lima, p. 63-99.

Florio, J 2008, "Desarrollo Sostenible de la Ganadería de Doble Propósito, Capítulo X. Uso de los bovinos criollos en cruzamientos con otras razas bovinas en América Latina, con énfasis en Ganadería Doble Propósito (en línea)", Venezuela. Consultado 03 nov. 2014, p. 116-126, Disponible en: http://www.avpa.ula.ve/libro_desarrollosost/pdf/capitulo_10.pdf

Henao, Fy Mejía, N 1994, "Barimetría en ganado Pardo Suizo", Rev Vet y Zoot Caldas, 7(4): 78- 81.

Hernández, M, García, F, Sotto, Vy López, M 1984, "Estimado del peso vivo al nacer en terneros cebú", Asociación Cubana de Producción Animal. Vol. 3, p. 42.

Mahecha, L, Angulo, J, Luis, P y Manrique, LP 2002a, "Predicción del peso vivo a través del perímetro torácico en la raza bovina Lucerna", Rev Col Cienc Pec. Vol. 15, pp. 88-91.

Mahecha, L, Angulo, J, y Manrique, LP 2002b, "Estudio bovinométrico y relaciones entre medidas corporales y el peso vivo en la raza Lucerna", Rev Col Cienc Pec., Vol. 15, pp. 80-87.

Matos, RR 1978, "Correlações entre medidas morfológicas e estimativas do desempenho de bovinos da raça Hereford submetidos a teste de avaliação", Anais XV Reunião Anual da Sociedad Brasileira de Zootecnia, Belem, Pará., pp. 232-236

Menéndez, B 1987, "Un método simple para evaluar hembras lecheras", Asociación Cubana de Producción Animal, vol. 3, pp. 13-19.

Muñoz, SA 2002, "Estadística aplicada uni y multivariante (2 volúmenes), Tomo I y II, Departamento de Genética", Universidad de Córdoba, España, pp. 01-1018.

Osorio, JCS, Vaz, CM, Jardim, P, Pimentel, M y Loguercio, A 1997, "Componentes do peso vivo na Raca Crioula", En: Congreso Brasileiro de Medicina Veterinaria, vol. 25. Gramado-Conbravet. pp. 266-268.

Quinto, CE 2004, "Inventario y capacidad de carga animal del Centro de Investigación de Camélidos Sudamericanos Lachocc", Tesis Ing. Zootecnista. Universidad Nacional de Huancavelica, Perú., pp. 01-101.

Ribeiro, MN, Da Silva, JV, Ribeiro, NL y Pimenta, EC 2000, "Correlation between body weight and body traits in native goats of Paraiba Estate, Brazil". In: global conference on CONSERVATION of domestic 
animal genetic resources, Brasília. Proceedings Brasília: RBI-Brazil. CDROM., pp. 09-15.

Ribeiro, MN, Da Silva, JV, Pimenta, EC y Sereno, JRB 2004, "Estudio de las correlaciones entre características fenotípicas de caprinos naturalizados", Arch. Zootec, Vol.53: pp. 337-340.
Rojas, R y Gómez, N 2005, "Biometría y constantes clínicas del bovino Criollo en el centro de investigación y producción Chuquibambilla de Puno (Perú)", Arch. Zootec. Vol. 54: pp. 233-236.

SAS 2009. "SAS/STAT: user's Guide: Statistics. Release 9.2", SAS Institute Inc. Cary, NC. 